Working Paper 94-54

Economics Series 26

December 1994
Departamento de Economía

Universidad Carlos III de Madrid

Calle Madrid, 126

28903 Getafe (Spain)

Fax (341) 624-9875

\title{
ON CONVERGENCE IN ENDOGENOUS GROWTH MODELS
}

Salvador Ortiguera and Manuel Santos*

\begin{abstract}
In this paper we analyze the rate of convergence to a balanced path in a class of endogenous growth models with physical and human capital. We show that such rate depends locally on the technological parameters of the model, but does not depend on those parameters related to preferences. These results stand in sharp contrast with those of the one-sector neoclassical growth model where both preferences and technologies determine the speed of convergence toward a steady state.
\end{abstract}

Key words: Neoclassical Growth Model, Endogenous Growth Models, Stability, Speed of Convergence.

- Departamento de Economía, Universidad Carlos III de Madrid and Centro de Investigación Económica, ITAM. Mexico D.F. 


\section{Introduction}

In this paper we analyze the speed of convergence to a balanced path in a class of endogenous growth models with physical and human capital. This class of models -initiated in the work of Uzawa (1965) and Lucas (1988)-- has been the focus of some current research in growth theory since they generate processes of permanent growth propelled by a human capital technology. [For a recent account of this literature, see Ladrón-de-Guevara et al. (1994).]

The speed of convergence provides important information in testing a model on the relative emphasis that should be placed on the steady-state behavior and transitional dynamics. If the speed of convergence to a steady state or balanced path is high, then the long-run behavior of the model should be determined by its predictions at the steady state. However, if such rate of convergence is low, then transitional dynamics may play a relevant role in ascertaining the predictive power of a model even if long-run considerations are called into the analysis.

Most recent studies have documented relatively low rates of convergence in both levels and rates of growth [e.g., see Barro (1991), Bernard and Durlauf (1992), Mankiw et al. (1993), and references therein]. Low rates of convergence are also confirmed from simple simulations of underlying economic growth models [e.g., King and Rebelo (1993) and Mulligan and Sala-i-Martin (1993)]. Although further research is needed to clarify some crucial issues in this area, what seems to emerge from this line of inquiry is that the transitional period may be quite long, and that countries may enjoy protracted episodes of high growth rates before they settle down to the steady-state behavior. Likewise, real shocks or government policies that lead to deviations from a steady-state growth path may be effective in changing the rate of growth over an extended time period.

In the neoclassical, one-sector growth model, the rate of convergence to a steady state is given by the smallest characteristic root of a quadratic polynomial corresponding to the linearization of Euler's equation. In such simple setting, this root can be computed by purely analytical methods. Moreover, one can further see from these computations the 
role played by different parameters of the model related to its technology and preferences.

In model economies with many types of capital goods, analytical methods may become unfeasible as the characteristic polynomial stemming from Euler's equations is of higher degree. The route suggested here is to proceed in such situations with a direct manipulation of the Euler equations, exploiting the basic idea that the smallest roots correspond to the eigenvalues of the derivative of the policy function [cf. Santos (1991), Prop. 2.3]. We should note in advance, however, that this simple procedure is bound not to be useful in general, ${ }^{1}$ but may be effective for the analysis of some aggregate models as those considered in this paper.

In our stylized class of endogenous growth models we then find that the rate of convergence depends in a quasilinear fashion on the technological parameters of the model. Roughly, this rate increases with the productivity of the human capital technology, and decreases with the productivity of physical capital in the goods sector. Population growth has a positive effect in the convergence rate to the extent that it acts as an added parameter in the depreciation of average physical capital. Remaining parameters embodied in the objective function concerning intertemporal utility for consumption and leisure have no effect on this rate. Thus, key variables of the model such as the discount rate, the relative weight of leisure on instantaneous utility or the elasticity of intertemporal substitution exert no influence in the speed at which an economy may approach a steady state.

The fact that preferences are ineffective in the determination of the speed of convergence seems to be related to the two-sectorial structure of our economies. In the one-sector growth model, the optimal quantities of labor and investment are determined by their shadow prices and the marginal utilities of leisure and consumption, respectively. In our two-sector framework with physical and human capital, the optimal amount of labor devoted to goods production depends on relative prices and productivities extant in the production and educational sectors. Furthermore, the evolution of relative prices must follow certain arbitrage identities in such a way that in some instances the equilibrium

\footnotetext{
${ }^{1}$ In some cases the characteristic roots may depend in a rather complex way on the parameters of the model; as a result, algebraic manipulations may become cumbersome.
} 
law of motion of labor is not directly affected by preferences parameters. We shall show that this somewhat neutral behavior of relative prices in the transition to a steady state holds true under fairly simple assumptions on utility functions and technologies.

Our study should be useful in guiding empirical work and numerical simulations. Although our analytical results are only valid for a neighborhood of the steady state, some numerical computations will illustrate that these estimates trace reasonably well the global (non-linear) converging behavior. Likewise, we would like to emphasize that for expositional convenience we proceed throughout the paper with the most basic technologies. The reader should bear in mind, however, that generally our models do not feature close-form solutions, and that our analytical methods extend to more general production functions.

The paper is structured as follows. We begin in Section 2 with a review of the onesector growth model where we illustrate our method of analysis. Then in Section 3 we present our main results on convergence for a family of two-sector growth models with qualified leisure. In Section 4 we show that our results are robust to several flat-rate distortionary taxes, leading to the conclusion that in some important instances fiscal policies may change steady state levels but become ineffective in altering rates of convergence to these solutions. In Section 5 we discuss two further extensions of the basic framework. The first model includes physical capital in the production of education, and the second one presents an alternative modelization of leisure. These two exercises are meant to shed light on major hypotheses underlying our results. Finally, we conclude in Section 6 with a summary of our main findings. The proofs of some basic assertions follow in a short appendix.

\section{The Neoclassical Growth Model}

In this section we present a simple version of the neoclassical growth model, and review some well known results on convergence. This setting will also prove useful to illustrate our approach in the following section.

We consider an economy where at each time $t \geq 0$ the production of the single homo- 
geneous good is represented by the production process

$$
y(t)=A k(t)^{\beta}
$$

where both variables $y(t)$ and $k(t)$ are measured in per capita units, and $A>0$ and $0<\beta<1$ are technological parameters. Output, $y(t)$, is devoted either to consumption, $c(t)$, or to investment, $i(t)$. Physical capital, $k(t)$, depreciates at a fixed rate, $\pi \geq 0$. The instantaneous utility derived from consumption is represented by a CES function

$$
U(c(t))=\frac{c(t)^{1-\sigma}-1}{1-\sigma}
$$

with $\sigma>0$. Future utilities are discounted at a given rate, $\rho>0$, and population grows at an exogenous rate, $n \geq 0$.

Under these assumptions, the planning problem can be written as

$$
\max \int_{0}^{\infty} e^{-(\rho-n) t} \frac{c(t)^{1-\sigma}-1}{1-\sigma} d t
$$

subject to

$$
\begin{aligned}
& \dot{k}(t)=A k(t)^{\beta}-(\pi+n) k(t)-c(t) \\
& c(t) \geq 0, \quad k(t) \geq 0 \\
& k(0)=k_{0} \text { given, } \rho-n>0
\end{aligned}
$$

where $\dot{k}(t)$ is the time derivative. It is well known that problem (P) has a unique, differentiable solution $\{(c(t), \dot{k}(t))\}_{t \geq 0}$, which must satisfy at every $t \geq 0$ the following system of first-order conditions

$$
\begin{array}{rlc}
c(t)^{-\sigma} & =c \\
\dot{\eta}_{1}(t) & =\left[\rho+\pi-\beta A k(t)^{\beta-1}\right] \eta_{1}(t)
\end{array}
$$

Here $\eta_{1}(t)$ denotes the co-state variable associated to $k(t)$. The optimal solution is characterized by (2.1)-(2.3) and the transversality condition,

$$
\lim _{t \rightarrow \infty} e^{-(\rho-n) t} \eta_{1}(t) k(t)=0
$$


From (2.1)-(2.3) we then obtain that the following two-dimensional dynamical system determines the evolution of consumption and investment,

$$
\begin{aligned}
& \dot{c}(t)=-\frac{c(t)}{\sigma}\left[\rho+\pi-\beta A k(t)^{\beta-1}\right] \\
& \dot{k}(t)=A k(t)^{\beta}-(\pi+n) k(t)-c(t)
\end{aligned}
$$

The system reaches a steady state if

$$
\begin{array}{rlc}
\rho+\pi & = & \beta A k^{k^{\beta-1}} \\
c^{*} & =A k^{* \beta}-(\pi+n) k^{*}
\end{array}
$$

It is easy to see from (2.7) and (2.8) that such a steady state $\left(c^{*}, k^{*}\right)$ is unique, and is given by the values

$$
c^{*}=A\left(\frac{\rho+\pi}{\beta A}\right)^{\frac{\beta}{\beta-1}}-(\pi+n)\left(\frac{\rho+\pi}{\beta A}\right)^{\frac{1}{\beta-1}}
$$

and

$$
k^{*}=\left(\frac{\rho+\pi}{\beta A}\right)^{\frac{1}{\beta-1}}
$$

In order to study the stability properties of the system, we linearize (2.5) and (2.6) at the steady-state values $\left(c^{*}, k^{*}\right)$. The linearized dynamical system is thus given by

$$
\left(\begin{array}{c}
\dot{c}(t) \\
\dot{k}(t)
\end{array}\right)=\left(\begin{array}{cc}
0 & -\frac{c^{*}}{\sigma}(1-\beta) \beta A k^{*} \\
-1 & \beta A k^{*-2} \\
-\beta-1
\end{array}\right)\left(\begin{array}{c}
c(t)-c^{*} \\
k(t)-k^{*}
\end{array}\right)
$$

where $c^{*}$ and $k^{*}$ are taken from (2.9) and (2.10), respectively. The characteristic equation corresponding to this linear system is then

$$
\lambda^{2}-(\rho-n) \lambda-\frac{(1-\beta)(\rho+\pi)}{\sigma}\left[\left(\frac{\rho+\pi}{\beta}\right)-(\pi+n)\right]=0
$$

It follows that the smallest root $\lambda_{1}$ is negative and it can be computed as

$$
\lambda_{1}=\frac{\rho-n-\left((\rho-n)^{2}+\frac{4(1-\beta)(\rho+\pi)}{\sigma}\left[\left(\frac{\rho+\pi}{\beta}\right)-(\pi+n)\right]\right)^{1 / 2}}{2}
$$


Consequently, equations (2.5)-(2.6) contain a one-dimensional stable manifold. Moreover, one could show that only solutions $(c(t), k(t))$ belonging to such stable manifold satisfy conditions (2.1)-(2.4). Hence, the stable manifold is the set of optimal solutions to problem $(\mathrm{P})$.

We now illustrate our approach to the stability problem in the context of this basic model. We start with the simple observation that in computing a unique root, we need only focus on a single equation of the system, say equation (2.5). Also, since the set of solutions $(c, k)$ conforming the stable manifold is a $C^{1}$ curve, we have that in general $k$ is going to be a $C^{1}$ function of $c$ and vice versa. At the steady state the derivative of $c$ with respect to $k, \frac{d c^{*}}{d k^{*}}$, can be computed from the eigenvector of the root $\lambda_{1}$ belonging to the $2 \times 2$ matrix in (2.11). We thus have

$$
\dot{c}(t)=-\frac{c(t)}{\sigma}\left[\rho+\pi-\beta A k(t)^{\beta-1}\right]
$$

Differentiating this equation with respect to $c(t)$ and evaluating the derivatives at steady states values, we obtain

$$
\dot{c}(t)=\left[-\frac{c^{*}}{\sigma}(1-\beta) \beta A k^{*}-\frac{d k^{*}}{d c^{*}}\right]\left(c(t)-c^{*}\right)
$$

Since $\lambda_{1}$ is the negative root, it must hold true that

$$
\lambda_{1}=\left[-\frac{c^{*}}{\sigma}(1-\beta) \beta A k^{*}{ }^{\beta-2} \frac{d k^{*}}{d c^{*}}\right]
$$

Also, since $\frac{d k^{*}}{d c^{*}}$ is the inverse of the slope of the stable manifold, a direct computation of the corresponding eigenvector of the $2 \times 2$ matrix in (2.11) yields that $\frac{d c^{*}}{d k^{*}}=\rho-n-\lambda_{1}$. Hence,

$$
\lambda_{1}\left(\rho-n-\lambda_{1}\right)=-\frac{c^{*}}{\sigma}(1-\beta) \beta A k^{*} \beta-2
$$

Plugging in the steady-state values $c^{*}$ and $k^{*}$, we get the same characteristic polynomial in (2.12), with negative root $\lambda_{1}$.

Let $\hat{\lambda}=-\lambda_{1}$. We shall call $\hat{\lambda}$ the rate or speed of convergence to a steady state $\left(c^{*}, k^{*}\right)$. The relatively complex form in (2.13) suggests that further extensions of the model such as leisure in the utility function or many types of goods- may render the rate of 
convergence $\hat{\lambda}$ hard to compute analytically. In the above simple case, however, we can see from (2.13) how different parameters related to preferences and technologies affect the value $\hat{\lambda}$. Observe that preferences parameters $\rho$ and $\sigma$ have a non-negligible influence on $\hat{\lambda}$. Indeed, one easily sees from (2.13) that $\hat{\lambda}$ becomes unbounded as $\sigma$ approaches zero.

We now calculate the rate of convergence $\hat{\lambda}$ in two cases, which will serve as reference in our later study. We first consider our benchmark economy with parameter values

$$
\sigma=1.5, \rho=0.05, \beta=0.4, n=0.01, \pi=0.05, \quad A=1
$$

In this case, $\hat{\lambda}=0.0694$. Our second example just involves a simple variation of the preceding values where $\sigma=0.4$ and the remaining parameters stay unchanged. In this situation, $\hat{\lambda}=0.15$. One sees then that in the benchmark economy the speed of convergence $\hat{\lambda}$ is relatively low, but it increases substantially with decrements in $\sigma$.

In order to investigate how accurately this local analytical result approximates the global converging behavior, we now calculate numerically the stable manifold of the system in both examples. For such purpose, we just follow a simple numerical technique ${ }^{2}$ where the stable manifold is extended backwards from an arbitrarily small neighborhood of the steady state, $k^{*}$. (In such small neighborhood, the stable manifold is approximated by the linearized stable system.) Figure 1 displays the laws of motion of the linear and nonlinear systems. It can be observed that the linearized system mimics well the non-linear dynamic behavior over a significative range of the capital domain. Therefore, in both examples the local speed of convergence, $\hat{\lambda}$, is a good estimate of the global converging behavior.

\section{An Endogenous Growth Model}

We now present a parameterized family of endogenous growth models with physical and human capital, and analyze the rate of convergence. With respect to the exogenous growth

\footnotetext{
${ }^{2}$ Our computations are effected by a standard Euler method [see, e.g., Gerald and Wheatley (1990, Ch. 5)].
} 
framework, this class of economies features an added educational sector and a choice of a time variable allocated to three margins: production of the aggregate good, schooling, and leisure activities.

At every time $t \geq 0$, production of the single, homogeneous good is represented by the production process

$$
y(t)=A k(t)^{\beta}(u(t) h(t))^{1-\beta}
$$

where $u(t)$ connotes the relative amount of effort devoted to the production of the good, and $h(t)$ is the level of education or human capital. All variables are measured in per capita units. In the educational sector, the law of motion of $h(t)$ is given by a linear technology

$$
\dot{h}(t)=\delta(1-u(t)-l(t)) h(t)-\theta h(t)
$$

where $(1-u(t)-l(t))$ is the fraction of time devoted to education, and $l(t)$ is the fraction of time spent in leisure activities. Parameter $\theta \geq 0$ is the rate of depreciation of $h(t)$, and parameter $\delta>0$ is the marginal productivity.

The instantaneous utility derived from consumption and leisure is represented by a CES function

$$
U(c, l h)=\frac{\left(c^{\gamma}(l h)^{1-\gamma}\right)^{1-\sigma}-1}{1-\sigma} \quad \text { if } \sigma>0, \sigma \neq 1,0<\gamma \leq 1
$$

and

$$
U(c, l h)=\gamma \ln c+(1-\gamma) \ln (l h) \quad \text { if } \quad \sigma=1,0<\gamma \leq 1
$$

Observe that for $\gamma=1$ this formulation reduces to the utility function postulated in Lucas (1988), and $\gamma=1$ and $\sigma=0$ is the case of linear preferences studied in Uzawa (1965).

The planning problem to be considered is written as

$$
V(k(0), h(0))=\max \int_{0}^{\infty} e^{-(\rho-n) t} \frac{\left(c(t)^{\gamma}(l(t) h(t))^{1-\gamma}\right)^{1-\sigma}-1}{1-\sigma} d t
$$

subject to

$$
\dot{k}(t)=A k(t)^{\beta}(u(t) h(t))^{1-\beta}-(\pi+n) k(t)-c(t)
$$




$$
\begin{aligned}
& \dot{h}(t)=\delta(1-u(t)-l(t)) h(t)-\theta h(t) \\
& 0 \leq u(t) \leq 1,0 \leq l(t) \leq 1,0 \leq u(t)+l(t) \leq 1, \\
& c(t) \geq 0, k(t) \geq 0, h(t) \geq 0 \\
& k(0), h(0) \text { given, } \rho-n>(\delta-\theta)(1-\sigma)
\end{aligned}
$$

Under these assumptions, problem $\left(P^{\prime}\right)$ has a unique optimal solution $\{(c(t), k(t), h(t)$, $l(t), u(t))\}_{t \geq 0}$, which in the interior case must satisfy the following system of first order conditions

$$
\begin{gathered}
\gamma c(t)^{(1-\sigma) \gamma-1}(l(t) h(t))^{(1-\sigma)(1-\gamma)}=\eta_{1}(t) \\
(1-\gamma) c(t)^{(1-\sigma) \gamma}(l(t) h(t))^{-\sigma-(1-\sigma) \gamma}=\eta_{2}(t) \delta \\
\eta_{1}(t)(1-\beta) A k(t)^{\beta}(u(t) h(t))^{-\beta}=\eta_{1}(t) \delta \\
\dot{\eta}_{1}(t)=\left[\rho+\pi-\beta A k(t)^{\beta-1}(u(t) h(t))^{1-\beta}\right] \eta_{1}(t) \\
\dot{\eta}_{2}(t)=[\rho-n-\delta+\theta] \eta_{2}(t)
\end{gathered}
$$

where $\eta_{1}(t)$ and $\eta_{2}(t)$ denote the co-state variables associated to $k(t)$ and $h(t)$, respectively. The optimal solution must also fulfil the transversality condition,

$$
\lim _{t \rightarrow \infty} e^{-(\rho-n) t}\left(\eta_{1}(t) k(t)+\eta_{2}(t) h(t)\right)=0
$$

A balanced path is an optimal solution $\left\{\left(c(t)^{*}, k(t)^{*}, h(t)^{*}, l(t)^{*}, u(t)^{*}\right)\right\}_{t \geq 0}$ to $\left(P^{\prime}\right)$ such that $c(t)^{*}, k(t)^{*}$ and $h(t)^{*}$ grow at constant rates and $l(t)^{*}$ and $u(t)^{*}$ stay constant. It follows from Caballe and Santos (1993) and Ortigueira (1994) that there is a unique ray of balanced paths, which is globally stable. Along this ray, consumption, $c(t)^{*}$, and both types of capital, $k(t)^{*}$ and $h(t)^{*}$, grow at a constant rate, say $\nu$.

Let $z(t)=\frac{c(t)}{k(t)}$ and $x(t)=\frac{h(t)}{k(t)}$. Then the ray of balanced paths can be parameterized by the vector $\left(z^{*}, x^{*}, l^{*}, u^{*}\right)$, and such vector remains invariant over the ray of balanced paths. Moreover, such a stationary solution, $\left(z^{*}, x^{*}, l^{*}, u^{*}\right)$, is characterized by 
the following system of equations

$$
\begin{array}{ccc}
\frac{1-\gamma}{\gamma} & = & \frac{l}{z u}(1-\beta) A x^{1-\beta} u^{1-\beta} \\
\rho+\sigma \nu+\pi & = & \beta A x^{1-\beta} u^{1-\beta} \\
\rho-n+\sigma \nu+\theta & = & \delta \\
z & = & A x^{1-\beta} u^{1-\beta}-\nu-n-\pi \\
\nu & = & \delta(1-u-l)-\theta
\end{array}
$$

Equation (3.6) is obtained after some simple arrangements from (3.1)-(3.3). Equations (3.7)-(3.8) are the corresponding versions of (3.4)-(3.5), and conditions (3.9)-(3.10) come from the technological laws of motion specified in $\left(P^{\prime}\right)$.

We now present our basic result on the speed of convergence.

Theorem 3.1: Assume that $\left(z^{*}, x^{*}, l^{*}, u^{*}\right)$ is an interior stationary solution to problem $\left(P^{\prime}\right)$. Then every other optimal arbitrary solution $(z(t), x(t), l(t), u(t))$ approaches $\left(z^{*}, x^{*}, l^{*}, u^{*}\right)$ at the rate of convergence,

$$
\hat{\lambda}=(1-\beta)\left(\frac{\delta+n+\pi-\theta}{\beta}\right)
$$

As in Caballé and Santos (1993), the ray of steady states could alternatively be parameterized by the variables $\tilde{k}(t)=k(t) e^{-\nu t}$ and $\tilde{h}(t)=h(t) e^{-\nu t}$, where $\nu$ is the steadystate growth rate. One can show that in such situation, every arbitrary optimal solution $(\tilde{k}(t), \tilde{h}(t))$ approaches a vector of steady state values $\left(\tilde{k}^{*}, \tilde{h}^{*}\right)$ at the same rate, $\hat{\lambda}$.

The most striking feature of the rate of convergence, $\hat{\lambda}$, is its simple, quasilinear dependence on parameter values. Moreover, in contrast to our parallel analysis of the one-sector growth model, we can see that preference parameters - which are generally associated in most calibrations with high standard errors- have no bearing on the rate $\hat{\lambda}$. We should note, however, that there are no well-known, independent estimates of educational productivity parameter, $\delta$, and its values are usually obtained from observations related to the model.

As shown in the appendix [see (7.6) below] an alternative expression for the rate of 
convergence is

$$
\hat{\lambda}=F_{L}\left(1, u^{*} x^{*}\right) u^{*} x^{*}
$$

where $F_{L}\left(1, u^{*} x^{*}\right)$ denotes the derivative of the production function $F(k, u h)$ with respect to the second argument, $L=u h$, at the steady state value $\left(1, u^{*} x^{*}\right)$. This expression remains valid in the more general case in which $F(k, L)$ is merely a linearly homogeneous function in $k$ and $L$. Furthermore, under this homogeneity assumption we obtain an equivalent formulation in terms of the marginal productivity of physical capital. That is,

$$
\hat{\lambda}=F\left(1, u^{*} x^{*}\right)-F_{k}\left(1, u^{*} x^{*}\right)=\frac{F\left(k^{*}, u^{*} h^{*}\right)}{k^{*}}-F_{k}\left(k^{*}, u^{*} h^{*}\right)
$$

for steady state levels $\left(k^{*}, u^{*} h^{*}\right)$. It is worth emphasizing that all these results are also heavily dependent on the homogeneity of degree one of the human capital technology in the amount of qualified labor employed in that sector, $(1-u-l) h$. If for instance the human capital technology is characterized by a production function of the form $G(1-u-l) h$, where $G(\cdot)$ is an increasing concave function with second order derivative $G^{\prime \prime}(\cdot)<0$, then the above results are no longer valid, since in such case production function $G(1-u-l) h$ is not linearly homogeneous in $(1-u-l) h$.

In order to compare our results with those of the preceding section, we again compute the rate of convergence in two cases. Our benchmark economy in this section has the following parameter values

$$
\delta=0.07, \gamma=0.45, \sigma=1.5, \rho=0.05, \beta=0.4, \quad n=0.01, \pi=0.05, \quad \theta=0, A=3
$$

These parameter values give rise to a steady state rate of growth, $\nu=0.02$. The speed of convergence is $\hat{\lambda}=0.1950$.

In order to obtain a close parallel of the exogenous growth framework, we now abstract from leisure considerations in the utility function and thus let $\gamma=1$. Observe that this parameterization results in the utility function set forth in Lucas (1988), which is also included under our general formulation. Moreover, if $\sigma=\beta$ then in this case the time devoted to education remains locally constant over the optimal solution [see Caballe and Santos (1993)], and hence the transitional dynamics are qualitatively the same as those 
of the neoclassical growth model. We then fix $\gamma=1, \sigma=\beta=0.4, \delta=0.05$, and leave unchanged the remaining parameter values of our benchmark economy. (Parameter $\delta$ has been adjusted in order to obtain an interior steady state with a more realistic time allocation for work and education.) This calibration yields a steady state growth rate $\nu=0.025$, and a speed of convergence $\hat{\lambda}=0.165$. Notice that the small difference in the rate of convergence with respect to the similar calibration in the preceding section (where $\hat{\lambda}=0.15)$ is due to the fact that for the postulated parameter values the rate of growth is now $\nu=0.0250$, whereas in the preceding section, $\nu=0$.

From these numerical exercises we can see that the speed of convergence differs substantially over the two benchmark cases (in the preceding section the benchmark value, $\hat{\lambda}=0.0694$, whereas in this section, $\hat{\lambda}=0.1950$ ). Furthermore, as it is evident from the foregoing calculations, in order to equate both rates of convergence parameter $\sigma$ must take values near 0.4 in the exogenous growth model.

Following our earlier numerical procedures, Figure 2 portrays the global dynamics of the linear and non-linear systems for our benchmark economies with endogenous growth. Vie again see that in both cases the linear system mimics reasonably well the non-linear converging behavior of the optimal solution over a sizable domain of state variable $\frac{k}{h}$. Simjlar results were obtained for various alternative parameterizations of the model. Hence, it appears that the same dynamic forces prevail over substantial phases of the transition with the effect that expressions (3.11)-(3.13) are good global estimates of the speed at which an economy will reach the steady state behavior.

\section{Fiscal Policy and Convergence}

We next apply our analysis to competitive economies with tax distortions. We consider that consumption, investment and income from capital and labor are subject, respectjvely, to ad valorem taxes $\tau_{c}, \tau_{i}, \tau_{k}, \tau_{l}$. Total revenues from taxation are rebated in lump sum form to the representative consumer. Under these circumstances, we now outline how our previous framework can be turned into a decentralized economy. 
For given initial values $k_{0}$ and $h_{0}$, the representative consumer is confronted with maximizing the objective in $\left(P^{\prime}\right)$ subject to the instantaneous budget balance

$$
\left(1+\tau_{c}\right) c(t)+\left(1+\tau_{i}\right) i(t)=\left(1-\tau_{k}\right) r(t) k(t)+\left(1-\tau_{l}\right) \omega(t) u(t) h(t)+T(t)
$$

and the laws of accumulation for the capital stocks,

$$
\begin{aligned}
& \dot{k}(t)=i(t)-(\pi+n) k(t) \\
& \dot{h}(t)=\delta(1-u(t)-l(t)) h(t)-\theta h(t)
\end{aligned}
$$

for all $t \geq 0$. Here, $i(t)$ denotes the rate of investment, $T(t)$ is the lump sum transfer to the agent, and $r(t)$ and $\omega(t)$ denote the rental prices for capital and labor, respectively.

For $t \geq 0$, the first-order conditions for the representative consumer are then

$$
\begin{aligned}
\gamma c(t)^{(1-\sigma) \gamma-1}(l(t) h(t))^{-(1-\sigma) \gamma+1-\sigma} & =\eta_{1}(t)\left(\frac{1+\tau_{c}}{1+\tau_{i}}\right) \\
(1-\gamma) c(t)^{(1-\sigma) \gamma}(l(t) h(t))^{-(1-\sigma) \gamma-\sigma} & =\eta_{2}(t) \delta \\
\eta_{1}(t)\left(\frac{1-\tau_{l}}{1+\tau_{i}}\right) \omega(t) & =\eta_{2}(t) \delta \\
\frac{\dot{\eta}_{1}(t)}{\eta_{1}(t)} & =\rho+\pi-\left(\frac{1-\tau_{k}}{1+\tau_{i}}\right) r(t) \\
\frac{\dot{\eta}_{2}(t)}{\eta_{2}(t)} & =\rho-n+\theta-\delta
\end{aligned}
$$

where $\eta_{1}(t)$ and $\eta_{2}(t)$ are the corresponding values for the co-state variables, which conform the path of prices for the competitive economy. The production sector is made up of a representative firm operating under the constant returns to scale technology, $y(t)=$ $A k(t)^{\beta}(u(t) h(t))^{1-\beta}$. The representative consumer rents capital and labor to the firm and these factors are paid according to the values of their marginal productivities. Also, in a competitive equilibrium, the goods and labor markets must clear. Therefore,

$$
\begin{aligned}
\omega(t) & =(1-\beta) k(t)^{\beta}(u(t) h(t))^{-\beta} \\
r(t) & =\beta k(t)^{\beta-1}(u(t) h(t))^{1-\beta}
\end{aligned}
$$

and

$$
y(t)=c(t)+i(t)
$$


for all $t \geq 0$. Imposing steady state conditions we obtain the following equations system

$$
\begin{array}{cccc}
\frac{1-\gamma}{\gamma} & = & \frac{l}{2 u}\left(\frac{1-\tau_{i}}{1+\tau_{c}}\right)(1-\beta) A x^{1-\beta} u^{1-\beta} \\
z & = & x^{1-\beta} u^{1-\beta}-\nu-n-\pi \\
\rho+\sigma \nu+\pi & = & & \left(\frac{1-\tau_{k}}{1+\tau_{i}}\right) \beta A x^{1-\beta} u^{1-\beta} \\
\rho-n+\sigma \nu+\theta & = & \delta \\
\nu & = & & \delta(1-u-l)-\theta
\end{array}
$$

The tax structure may affect the steady state values $z^{*}, x^{*}, l^{*}$ and $u^{*}$ but in our simple model one can see from (4.4) that it will not affect the growth rate $\nu$. We shall presently show that the rate of convergence to the new steady state is also unaffected by the presence of the distortionary fiscal policy. This result again differentiates our endogenous growth framework from the neoclassical model where under the same functional forms flat rate taxes on capital rents have a significant impact on the rate of convergence. Consequently, our analysis highlights the testable restriction that the rate of convergence is independent of the fiscal regime.

Following the same arguments as in our previous theorem the corresponding formulation of (3.12)-(3.13) is now

$$
\hat{\lambda}=(1-\beta)\left(\frac{1-\tau_{k}}{1+\tau_{i}}\right) A x^{*^{1-\beta}} u^{*^{1-\beta}}
$$

Making then use of equations (4.3) and (4.4) it is readily shown that

$$
\hat{\lambda}=(1-\beta)\left(\frac{\delta+n+\pi-\theta}{\beta}\right)
$$

This is, of course, expression (3.11) obtained in the preceding section.

\section{Extensions of the Basic Framework}

We now examine two further extensions of the previous framework. Our first economy includes physical capital as an added factor in the production of education. Assuming linear homogeneity of the technologies in both sectors, we again obtain that preference 
parameters are still ineffective in the determination of the speed of convergence. Our second economy is concerned with a diametrically opposite modelization of leisure, where human capital has no influence on its marginal productivity. This latter model may feature multiple balanced paths [see Ladrón-de-Guevara et al. (1994)]. In addition, we shall show below that preference parameters may affect marginally the speed of convergence.

\subsection{Physical Capital in the Human Capital Technology}

Models with physical capital for the production of education have been set forth in Bond, Wang and Yip (1992), Ladrón-de-Guevara et al. (1994), Mino (1992) and Mulligan and Sala-i-Martin (1993). It is known from this analysis that relative factor intensities play a major role in the dynamic evolution of economic variables along the transition to the unique ray of balanced paths, which is globally stable. For the sake of convenience, we shall confine our attention to what seems to be the more realistic case in which the educational sector is labor intensive. For this class of models, some numerical computations on the speed of convergence are discussed in Mulligan and Sala-i-Martin (1993). We shall provide here an analytical expression that comprises their findings.

To simplify matters, we assume that leisure considerations are absent in the choice theoretical problem of the economy. We then consider the following model

$$
\begin{aligned}
& V\left(k_{0}, h_{0}\right)=\max _{c(t), u(t), v(t)} \int_{0}^{\infty} e^{-(\rho-n) t} \frac{c(t)^{1-\sigma}}{1-\sigma} d t \quad\left(P^{\prime \prime}\right) \\
& \text { subject to } \\
& \dot{k}(t)=A[v(t) k(t)]^{\beta}[u(t) h(t)]^{1-\beta}-(\pi+n) k(t)-c(t) \\
& \dot{h}(t)=B[(1-v(t)) k(t)]^{\alpha}[(1-u(t)) h(t)]^{1-\alpha}-\theta h(t) \\
& c(t) \geq 0, k(t) \geq 0, h(t) \geq 0 \\
& 0 \leq u(t) \leq 1,0 \leq v(t) \leq 1 \\
& k_{0}, h_{0} \text { given, } \rho-n>0,0<\beta<1,0<\alpha<1
\end{aligned}
$$

As in previous cases, an interior optimal solution must obey the following system of first- 
order conditions

$$
\begin{aligned}
c(t)^{-\sigma} & =\eta_{1}(t) \\
\eta_{1}(t) \beta A[v(t) k(t)]^{\beta-1}[u(t) h(t)]^{1-\beta} & =\eta_{2}(t) \alpha B[(1-v(t)) k(t)]^{\alpha-1}[(1-u(t)) h(t)]^{1-\alpha} \\
\eta_{1}(t)(1-\beta) A[v(t) k(t)]^{\beta}[u(t) h(t)]^{-\beta} & =\eta_{2}(t)(1-\alpha) B[(1-v(t)) k(t)]^{\alpha}[(1-u(t)) h(t)]^{-\alpha} \\
\frac{\dot{\eta}_{1}(t)}{\eta_{1}(t)} & =\rho+\pi-\beta A\left[(v(t) k(t)]^{\beta-1}[u(t) h(t)]^{1-\beta}\right. \\
\frac{\dot{\eta}_{2}(t)}{\eta_{2}(t)} & =\rho-n+\theta-(1-\alpha) B[(1-v(t)) k(t)]^{\alpha}[(1-u(t)) h(t)]^{-\alpha}
\end{aligned}
$$

Let $x_{1}(t)=\frac{v(t) k(t)}{u(t) h(t)}$ and $x_{2}(t)=\frac{(1-v(t)) k(t)}{(1-u(t)) h(t)}$. Imposing steady-state conditions, we obtain that the unique optimal solution $\left(z^{*}, x_{1}^{*}, x_{2}^{*}, v^{*}, u^{*}, \nu\right)$ is characterized by the following equations system.

$$
\begin{array}{rlc}
u x A x_{1}^{\beta} & = & \pi+n+\nu+z \\
(1-u) B x_{2}^{\alpha} & = & \nu+\theta \\
\left(\frac{\beta}{1-\beta}\right) x_{2} & = & \left(\frac{\alpha}{1-\alpha}\right) x_{1} \\
\rho-n+\sigma \nu+\theta & = & (1-\alpha) B x_{2}^{\alpha} \\
\rho+\sigma \nu+\pi & = & \beta A x_{1}^{\beta-1}
\end{array}
$$

From (5.3), (5.4) and (5.5) we can solve for the steady state values $x_{1}, x_{2}$ and $\nu$; then, eq. (5.2) yields a unique value $u^{*}$. Likewise, given that $\frac{1}{x}=u x_{1}+(1-u) x_{2}$, we can find a solution for $z^{*}$ from eq. (5.1). Finally, from $x_{1}=\frac{v k}{u h}$ we obtain the steady state value for $v^{*}$. Hence, under these conditions there is at most a unique stationary solution $\left(z^{*}, x_{1}^{*}, x_{2}^{*}, v^{*}, u^{*}, \nu\right)$. It is known from the aforementioned references that such optimal solution is globally stable.

We now examine the rate of convergence about this solution. As already pointed out, we confine our analysis to the case in which the educational sector is intensive in human capital.

Theorem 5.1: Let $\alpha<\beta$. Assume that $\left(z^{*}, x_{1}^{*}, x_{2}^{*}, v^{*}, u^{*}\right)$ is an interior stationary solution to problem $\left(P^{\prime \prime}\right)$. Then every other optimal arbitrary solution approaches the steady state values $\left(z^{*}, x_{1}^{*}, x_{2}^{*}, v^{*}, u^{*}\right)$ at the rate of convergence, 


$$
\hat{\lambda}=\frac{(1-\beta) \beta A}{\beta-\alpha}\left(\frac{\alpha(1-\beta)}{\beta(1-\alpha)}\right)^{\frac{\alpha(\beta-1)}{\beta-\alpha}}\left(\frac{x_{2}^{* \alpha}}{x_{1}^{* \beta}}\right)^{\frac{1-\beta}{\beta-\alpha}}+\frac{\alpha(1-\alpha) B}{\beta-\alpha}\left(\frac{\alpha(1-\beta)}{\beta(1-\alpha)}\right)^{\frac{\alpha}{\beta-\alpha}}\left(\frac{x_{2}^{* \alpha}}{x_{1}^{* \beta}}\right)^{\frac{-\alpha}{\beta-\alpha}}
$$

Again, expression (5.6) does not depend on preference parameters since from (5.3)(5.4) we obtain that the values $x_{1}^{*}$ and $x_{2}^{*}$ are resolved from the equations system

$$
\begin{aligned}
\beta A x_{1}^{\beta-1}-\pi & =(1-\alpha) B x_{2}^{\alpha}+n-\theta \\
(1-\alpha) \beta x_{2} & =(1-\beta) \alpha x_{1}
\end{aligned}
$$

One can show from (5.6) that the derivatives $\frac{\partial \hat{\lambda}}{\partial \beta}$ and $\frac{\partial \hat{\lambda}}{\partial \alpha}$ dot not have a well determined sign. Observe, however, that as $\alpha \rightarrow 0$ the constant returns to scale technology for the production of education converges to the linear technology of Section 3 , and as a result $\hat{\lambda}$ converges to expression (3.11) for $\delta=B$. Moreover, as $\beta \rightarrow 1$ then $\hat{\lambda} \rightarrow 0$, and as $\alpha \rightarrow \beta$ then $\hat{\lambda} \rightarrow \infty$. As noted in Mulligan and Sala-i-Martin (1993) if $\alpha \rightarrow \beta$ then the production possibility frontier involving both sectors becomes a straight line. Therefore, the absence of adjustment costs in shifting resources from one sector to the other gives rise to an infinite rate of convergence.

\subsection{Pure Leisure in the Utility Function}

We close our analysis with a polar modelization of leisure. We assume now that human capital does not affect its marginal utility. The framework is the same as that in Section 3 , but the utility function is now written as follows

$$
\begin{aligned}
& U(c, l)=\frac{\left(c^{\gamma} l^{1-\gamma}\right)^{1-\sigma}-1}{1-\sigma} \text { for } \sigma>0, \sigma \neq 0 \text { and } 0<\gamma \leq 1 \\
& U(c, l)=\gamma \log c+(1-\gamma) \log l, \text { for } \sigma=1
\end{aligned}
$$

This model is considered in Lucas (1990) and Ladrón-de-Guevara et al. (1994). The rationale behind this formulation is that the utility obtained from certain leisure activities -such as sleeping or spending time with the family - does not depend upon the attained 
level of education. Ladrón-de-Guevara et al. (1994) show that for certain parameterizations this model may contain multiple steady state equilibria. For our present illustrative purposes, we shall focus on a calibrated economy with a unique balanced path and verify that changes in preferences parameters $\sigma$ and $\gamma$ will lead to marginal variations in the speed of convergence. At this stage, it is worth pointing out that as $\gamma$ converges to one, the utility function converges to a simple form included in the analysis of Section 3 , and accordingly in such a case preference parameters have no effect in the convergence rate.

In this new setting, we now assign the following parameter values

$$
\delta=0.2, \gamma=0.4, \sigma=1.5, \rho=0.05, \beta=0.4, n=0.01, \pi=0.05, \theta=0.05, A=3
$$

This model gives rise to a stationary growth rate $\nu=0.0229$. The speed of convergence about such stationary solution is $\hat{\lambda}=0.1908$. Starting from this reference model, we now vary separately parameters values $\sigma$ and $\gamma$. Table 1 displays the computed rate of convergence in the present model for different calibrations of $\sigma$ against the estimated values of the rate of convergence from analytic expression (3.12) of Section 3. Table 2 reproduces the same results for several values of $\gamma$, letting $\sigma=1.5$ fixed. It is seen from this exercise that these changes result in marginal variations from the estimated rate of convergence.

\begin{tabular}{|l|l|l|}
\hline & $\begin{array}{l}\text { true value } \\
\hat{\lambda}\end{array}$ & $\begin{array}{l}\text { estimated value } \\
F_{L}\left(1, u^{*} x^{*}\right) u^{*} x^{*}\end{array}$ \\
\hline$\sigma=2$ & .1892 & .1840 \\
\hline$\sigma=1.85$ & .1895 & .1857 \\
\hline$\sigma=1.75$ & .1897 & .1871 \\
\hline$\sigma=1.65$ & .1901 & .1886 \\
\hline$\sigma=1.5$ & .1908 & .1913 \\
\hline$\sigma=1.25$ & .1931 & .1976 \\
\hline$\sigma=1$ & .1980 & .2077 \\
\hline
\end{tabular}

Table 1. True and estimated values for the rate of convergence. 


\begin{tabular}{|l|l|l|}
\hline & $\begin{array}{l}\text { true value } \\
\hat{\lambda}\end{array}$ & $\begin{array}{l}\text { estimated value } \\
F_{L}\left(1, u^{*} x^{*}\right) u^{*} x^{*}\end{array}$ \\
\hline$\gamma=1$ & .3150 & .3150 \\
\hline$\gamma=.6$ & .2500 & .2477 \\
\hline$\gamma=.55$ & .2384 & .2364 \\
\hline$\gamma=.5$ & .2251 & .2236 \\
\hline$\gamma=.45$ & .2096 & .2089 \\
\hline$\gamma=.4$ & .1908 & .1913 \\
\hline$\gamma=.35$ & .1668 & .1693 \\
\hline
\end{tabular}

Table 2. True and estimated values for the rate of convergence.

\section{Concluding Remarks}

In this paper we have considered a family of endogenous growth models with physical and human capital. This framework seems potentially attractive to growth theorists, since it allows for the possibility of convergence in growth rates while preserving diferences in income levels. Moreover, in response to imbalances in the ratio of physical to human capital this class of models features a rich dynamic behavior in the process of convergence to the steady state path.

Our study has focused on the speed of convergence to the steady state path. A few testable propositions have emerged from this analysis. Our principal finding in Section 3 is a neat characterization of the local rate of convergence. The result asserts that the productivity of the human capital technology has a positive influence on the speed of convergence, while the income share of physical capital exhibits the reverse effect. Moreover, key parameter values of the model concerning preferences and distortionary taxes exert no influence on the rate at which an economy will approach the steady state path.

These findings may be contrasted with an analogous analysis of the exogenous growth model, where preference data as well as flat-rate taxes on capital rentals play a significant 
role in assessing the speed of convergence. Besides, our numerical computations attest that for calibrated parameter values the endogenous growth model displays a substantially higher rate of convergence over relevant phases of the transition. In order for the neoclassical growth model to exhibit the same rate of convergence, the inverse of the elasticity of intertemporal substitution, $\sigma$, must roughly match the value of the elasticity of labor with respect to physical capital, $\beta$. Since standard calibrations of $\beta$ are generally below 0.5 , this equality would result in rather implausible values for $\sigma$. For our benchmark economies, a halving of the stock of physical capital entails roughly a forty-year transition period in the exogenous growth model in order for such stock to lie within a one-percent distance of the original level. Such transitional process will approximately involve a tweenty-three-year period in the endogenous growth model.

Of course, a thorough study of the dynamics in these two models would examine the transitional behavior of individual variables such as consumption, physical and human capital, leisure, and work. Such detailed investigation would shed light on other aspects of the process of convergence, and would complement the analysis of most of the abovementioned references. Yet, this endeavor was not the original purpose of the present paper.

The above results hold true for basic functional forms on utilities and technologies. Such functional forms are generally hypothesized in empirical applications. It appears from our analysis in Section 5, however, that the most fundamental properties underlying these results are linear homogeneity of the production functions and convexity of the set of optimal decisions. ${ }^{3}$ Also, our numerical computations in that section illustrate that deviations from these fundamental properties may result in marginal departures from our benchmark estimates.

Further research is, of course, needed in order to gain a better understanding of these issues. Care in the elaboration of data series on human capital, taxes, and other relevant

\footnotetext{
${ }^{3}$ The model with pure leisure in the utility function of Section 5 cannot be associated with a concave optimization problem, since letting $h_{m}=m h$, for $m=l, u,(1-u-l)$, this formulation may be rewritten as a concave optimization problem with an added negative externality of aggregate human capital, $h$, in the utility function.
} 
variables may help in discriminating among competing families of models. Likewise, theoretical work along the directions pursued here should lead to sharper tests of convergence. 


\section{Appendix}

Throughout this appendix we shall assume that the one-dimensional stable manifold lies in a general position, so that it can be parameterized by each of the coordinates $\left(c, k, h, l, u, \eta_{1}, \eta_{2}\right)$. One can show from the analysis of Caballe and Santos (1993) and Ortigueira (1994) that this property must hold generically. Moreover, if a property on the value of a characteristic root holds true for a generic subset of matrices, by continuity such property must hold true for the whole set of matrices.

In the proof of the theorem, we make use of the following lemma.

Lemma 1: Let $\frac{d x^{*}}{d u^{*}}$ be the derivative of $x$ with respect to $u$ at the steady state $\left(z^{*}, x^{*}, l^{*}, u^{*}\right)$. Then

$$
-\frac{d x^{*}}{d u^{*}} \frac{u^{*}}{x^{*}} \neq 1
$$

Proof: Takings logs in (3.3), making use of the fact that $\eta_{2}(t)$ grows at the constant rate $\rho-n-\delta+\theta$ over the optimal solution [see (3.5)], and differentiating with respect to $t$, we have

$$
\frac{\dot{\eta}_{1}(t)}{\eta_{1}(t)}-\beta \frac{\dot{x}(t)}{x(t)}-\beta \frac{\dot{u}(t)}{u(t)}=\rho-n-\delta+\theta
$$

Write $\dot{\eta}_{1}(t), \dot{x}(t)$ and $\dot{u}(t)$ as functions of their own variable. That is, $\dot{\eta}_{1}(t)=g\left(\eta_{1}(t)\right)$, $\dot{x}(t)=f(x(t))$ and $\dot{u}(t)=h(x(t))$. Then

$$
\frac{g\left(\eta_{1}(t)\right)}{\eta_{1}(t)}-\beta \frac{f(x(t))}{x(t)}-\beta \frac{g(u(t))}{u(t)}=\rho-n-\delta+\theta
$$

Considering that derivatives $g^{\prime}\left(\eta_{1}^{*}\right)=f^{\prime}\left(x^{*}\right)=h^{\prime}\left(u^{*}\right)=\lambda_{1}=-\hat{\lambda}$, we differentiate expression ( $(\pi .1)$ with respect to $x(t)$, and evaluate the derivatives at their steady state values. We then obtain

$$
\frac{d \eta_{1}^{*}}{d x^{*}}=\left[\frac{\beta}{x^{*}}+\frac{\beta \frac{d u^{*}}{d x^{*}}}{u^{*}}\right] \eta_{1}^{*}
$$

Assume now that

$$
\frac{d x^{*}}{d u^{*}} \frac{u^{*}}{x^{*}}=-1
$$

This implies

$$
\frac{d \eta_{1}^{*}}{d x^{*}}=\left[\frac{\beta}{x^{*}}-\frac{\beta}{x^{*}}\right] \eta_{1}^{*}=0
$$


We next show that (7.2) is in contradiction with the fact that the value function $V(k, h)$ for problem $\left(P^{\prime}\right)$ is a smooth concave function homogeneous of degree $1-\sigma$, with negative second-order own derivative.

Observe that

$$
\eta_{1}(t)=V_{k}(k(t), h(t))
$$

where $V_{k}(\cdot, \cdot)$ is the partial derivative of $V(\cdot, \cdot)$ with respect to $k(t)$. Moreover, notice that if we make the normalization $h=1$ then

$$
\frac{d \eta_{1}^{*}}{d x^{*}}=-V_{k k}\left(\frac{1}{x^{*}}, 1\right) \frac{1}{x^{*^{2}}}>0
$$

where $V_{k k}(\cdot, \cdot)$ is the second-order partial derivative of $V(\cdot, \cdot)$ with respect to $k(t)$. We now see that expression (7.2) is in contradiction with (7.3). The Lemma is proved.

Although we have shown in our model that $\frac{d x^{*}}{d u^{*}} x^{*}=-1$ is not compatible with the concavity of the value function $V(k, h)$, it should be remarked that this equality $\frac{d x^{*}}{d u^{*}} \frac{u^{*}}{x^{*}}=$ -1 is usually not robust to small perturbations of the parameter space. Under these latter circumstances, our method of proof remains still valid.

Proof of the Theorem 3.1: By the definition of $\hat{\lambda}$, for every arbitrarily small $\epsilon>0$, there is $T$ such that

for all $t \geq T$.

$$
\left|\frac{\dot{u}(t)}{u(t)-u^{*}}-\lambda_{1}\right|<\epsilon
$$

Taking logs in (3.3), making use of the fact that $\eta_{2}(t)$ grows at a constant rate [see (3.5)] and differentiating with respect to $t$, we get

$$
\frac{\dot{\eta}_{1}(t)}{\eta_{1}(t)}-\beta \frac{\dot{x}(t)}{x(t)}-\beta \frac{\dot{u}(t)}{u(t)}=\rho-n-\delta+\theta
$$

Substituting out $\frac{\dot{\eta}_{1}(t)}{\eta_{1}(t)}$ from (3.4) yields

$$
\dot{u}(t)=\left[\delta+n+\pi-\theta-\beta A x(t)^{1-\beta} u(t)^{1-\beta}-\beta \frac{\dot{x}(t)}{x(t)}\right] \frac{u(t)}{\beta}
$$

Linearizing $\dot{u}(t)$ around its steady-state value $u^{*}$, we get

$$
\dot{u}(t)=\left[-(1-\beta) A x^{x^{*}-\beta} u^{*^{1-\beta}}-(1-\beta) A x^{*-\beta} u^{*^{2-\beta}} \frac{d x^{*}}{d u^{*}}-\frac{d}{d u^{*}}\left(\frac{\dot{x}^{*}}{x^{*}}\right) u^{*}\right]\left(u(t)-u^{*}\right)
$$


Write $\dot{x}(t)=f(x(t))$, and observe that $f^{\prime}\left(x^{*}\right)=\lambda_{1}$. It follows that

$$
\dot{u}(t)=\left[-(1-\beta) A x^{*^{1-\beta}} u^{*^{1-\beta}}-(1-\beta) A x^{*-\beta} u^{*^{2-\beta}} \frac{d x^{*}}{d u^{*}}-\left(\frac{\lambda_{1}}{x^{*}} \frac{d x^{*}}{d u^{*}}\right) u^{*}\right]\left(u(t)-u^{*}\right)
$$

From (7.4) and (7.5) we then must have

$$
\lambda_{1}=-(1-\beta) A x^{*^{1-\beta}} u^{*^{1-\beta}}-\frac{d x^{*}}{d u^{*}}\left((1-\beta) A x^{*-\beta} u^{*^{2-\beta}}+\frac{\lambda_{1}}{x^{*}} u^{*}\right)
$$

Hence,

$$
\left[\lambda_{1}+(1-\beta) A x^{*^{1-\beta}} u^{*^{1-\beta}}\right]=\left[-\frac{d x^{*}}{d u^{*}} \frac{u^{*}}{x^{*}}\right]\left[\lambda_{1}+(1-\beta) A x^{*^{1-\beta}} u^{*^{1-\beta}}\right]
$$

Therefore, by Lemma 1 ,

$$
\lambda_{1}=-(1-\beta) A x^{*^{1-\beta}} u^{*^{1-\beta}}
$$

Going back to equations (3.7) and (3.8), we finally get

$$
\lambda_{1}=-(1-\beta)\left(\frac{\delta+n+\pi-\theta}{\beta}\right)
$$

The theorem now follows from our notational convention, $\hat{\lambda}=-\lambda_{1}$.

Proof of the Theorem 5.1: Define $p(t)$ as the relative price $\frac{\eta_{1}(t)}{\eta_{2}(t)}$. Then, from the above first order conditions we can write the dynamics of $p(t)$ as

$$
\begin{aligned}
\frac{\dot{p}(t)}{p(t)}= & \pi+n-\theta-\beta A\left(\frac{1-\beta}{1-\alpha}\right)^{\frac{(\alpha-1)(\beta-1)}{\beta-\alpha}}\left(\frac{\alpha}{\beta}\right)^{\frac{\alpha(\beta-1)}{\beta-\alpha}}\left(\frac{A}{B}\right)^{\frac{1-\beta}{\beta-\alpha}} p(t)^{\frac{1-\beta}{\beta-\alpha}}+ \\
& +(1-\alpha) B\left(\frac{1-\beta}{1-\alpha}\right)^{\frac{\alpha(\beta-1)}{\beta-\alpha}}\left(\frac{\alpha}{\beta}\right)^{\frac{\alpha \beta}{\beta-\alpha}}\left(\frac{A}{B}\right)^{\frac{-\alpha}{\beta-\alpha}} p(t)^{\frac{-\alpha}{\beta-\alpha}}
\end{aligned}
$$

Linearizing $\dot{p}(t)$ around $p^{*}$ we obtain

$$
\begin{aligned}
\lambda_{1}= & -\frac{(1-\beta) \beta A}{\beta-\alpha}\left(\frac{1-\beta}{1-\alpha}\right)^{\frac{(\alpha-1)(\beta-1)}{\beta-\alpha}}\left(\frac{\alpha}{\beta}\right)^{\frac{\alpha(\beta-1)}{\beta-\alpha}}\left(\frac{A}{B}\right)^{\frac{1-\beta}{\beta-\alpha}} p^{\frac{1-\beta}{\beta-\alpha}}- \\
& -\frac{(1-\alpha) \alpha B}{\beta-\alpha}\left(\frac{1-\beta}{1-\alpha}\right)^{\frac{\alpha(\beta-1)}{\beta-\alpha}}\left(\frac{\alpha}{\beta}\right)^{\frac{\alpha \beta}{\beta-\alpha}}\left(\frac{A}{B}\right)^{\frac{-\alpha}{\beta-\alpha}} p^{\frac{-\alpha}{\beta}-\alpha}
\end{aligned}
$$


From such conditions we also obtain the steady state value for $p$ as

$$
p^{*}=\frac{(1-\alpha) B x_{2}^{*^{\alpha}}}{(1-\beta) A x_{1}^{* \beta}}
$$

Substituting out this expression in (7.7) we get the asserted value for $\hat{\lambda}=-\lambda_{1}$. 


\section{References}

[1] Barro, Robert J., "Economic Growth in a Cross-Section of Countries," Quarterly Journal of Economics, May 1991, 106, 407-445.

[2] Bernard, Andrew B. and Durlauf, Steven N., "Convergence of International Output Movements," NBER Working Paper, 1991, no. 3717.

[3] Bond, Eric W., Wang Ping and Yip, Chong K., "A General Two-Sector Model of Endogenous Growth with Human and Physical Capital," Mimeo, The Pennsylvania State University, 1992.

[4] Caballé, Jordi and Santos, Manuel S., "On Endogenous Growth with Physical and Human Capital," Journal of Political Economy, December 1993, 101, 1042-1068.

[5] Gerald, Curtis F. and Wheatley, Patrick O., Applied Numerical Analysis, fourth edition. Addison-Wesley Publishing Company, 1990.

[6] King. Robert G. and Rebelo, Sergio T., "Transitional Dynamics and Economic Growth in the Neoclassical Model," American Economic Review, September 1993, 83, 908-931.

[7] Ladrón-de-Guevara, Antonio, Ortigueira, Salvador and Santos, Manuel S., "Equilibrium Dynamics in Two-Sector Models of Endogenous Growth," Forthcoming in Journal of Economic Dynamics and Control, 1994.

[8] Lucas, Robert, Jr., "On the Mechanics of Economic Development," Journal of Monetary Economics, July 1988, 22, 3-43.

[9] Lucas, Robert, Jr., "Supply Side Economics: An Analytical Review," Oxford Economic Papers, April 1990, 42, 293-317.

[10] Mankiw, Gregory, Romer, David and Weil, David, "A Contribution to the Empirics of Economic Growth," Quarterly Journal of Economics, May 1992, 107, 407-437. 
[11] Mulligan, Casey B. and Sala-i-Martin, Xavier, "Transitional Dynamics in Two-Sector Models of Endogenous Growth," Quarterly Journal of Economics, August 1993, CVIII, 740-773.

[12] Mino, Kazuo, "Analysis of a Two-Sector Model of Endogenous Growth with Capital Income Taxation," Mimeo, Tohoku University, 1992.

[13] Ortigueira, Salvador, "A Dynamic Analysis of an Endogenous Growth Model with Leisure," Mimeo, Universidad Carlos III de Madrid, 1994.

[14] Santos, Manuel S., "Smoothness of the Policy Function in Discrete Time Economic Models," Econometrica, September 1991, 59, 1365-1382.

[15] Uzawa, Hirofumi, "Optimum Technical Change in an Aggregate Model of Economic Growth," International Economic Review, January 1965, 6, 18-31. 


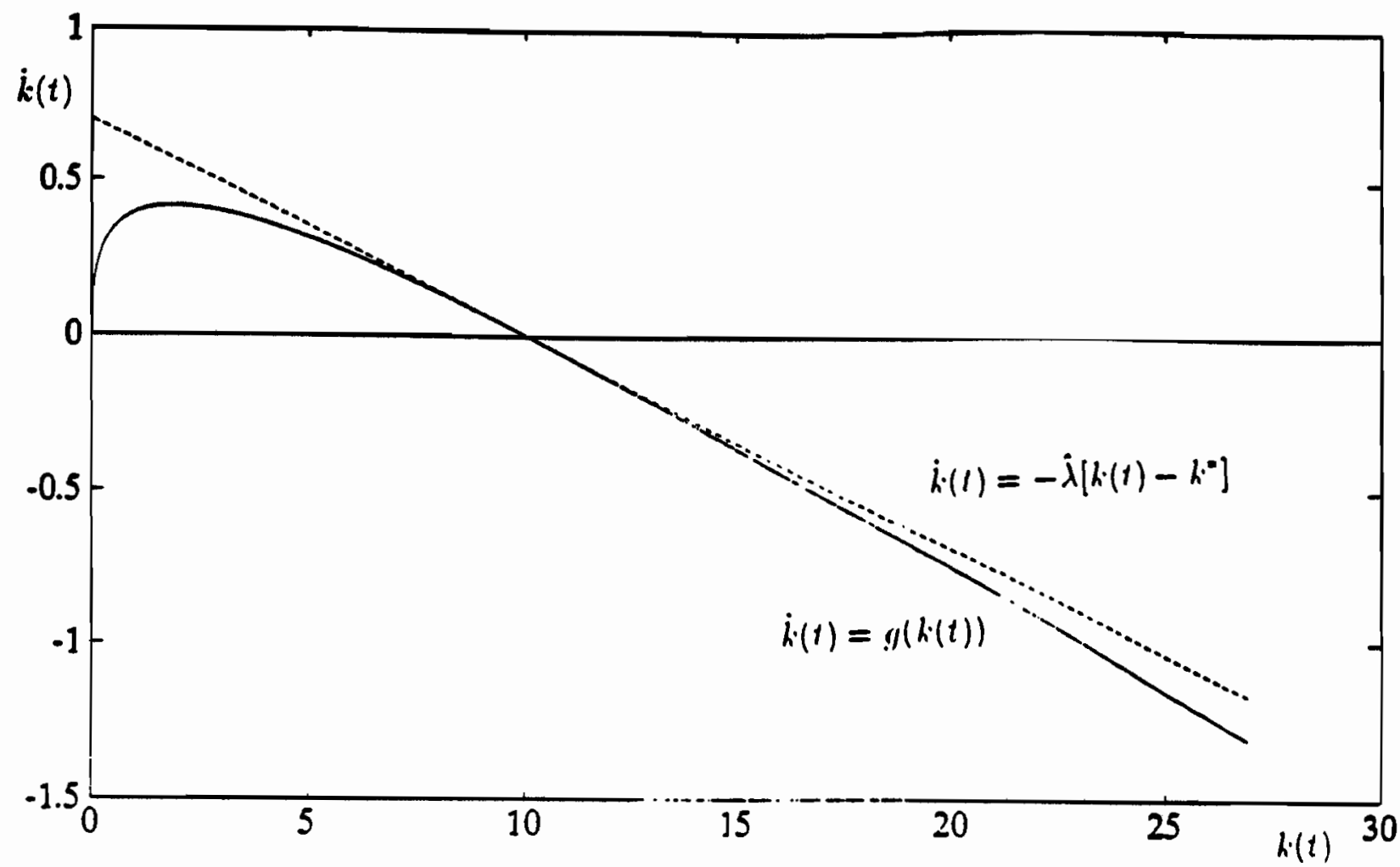

Figure 1(a). Dynamics in the Neoclassical Grouth Model: Stable manifolds of the linear and non-linear systems for state variable $k$. Parameter values, $\sigma=1.5, \rho=0.05$, $\beta=0.4, n=0.01, \pi=0.05, A=1$. Steady state, $k^{*}=10.0794$. 


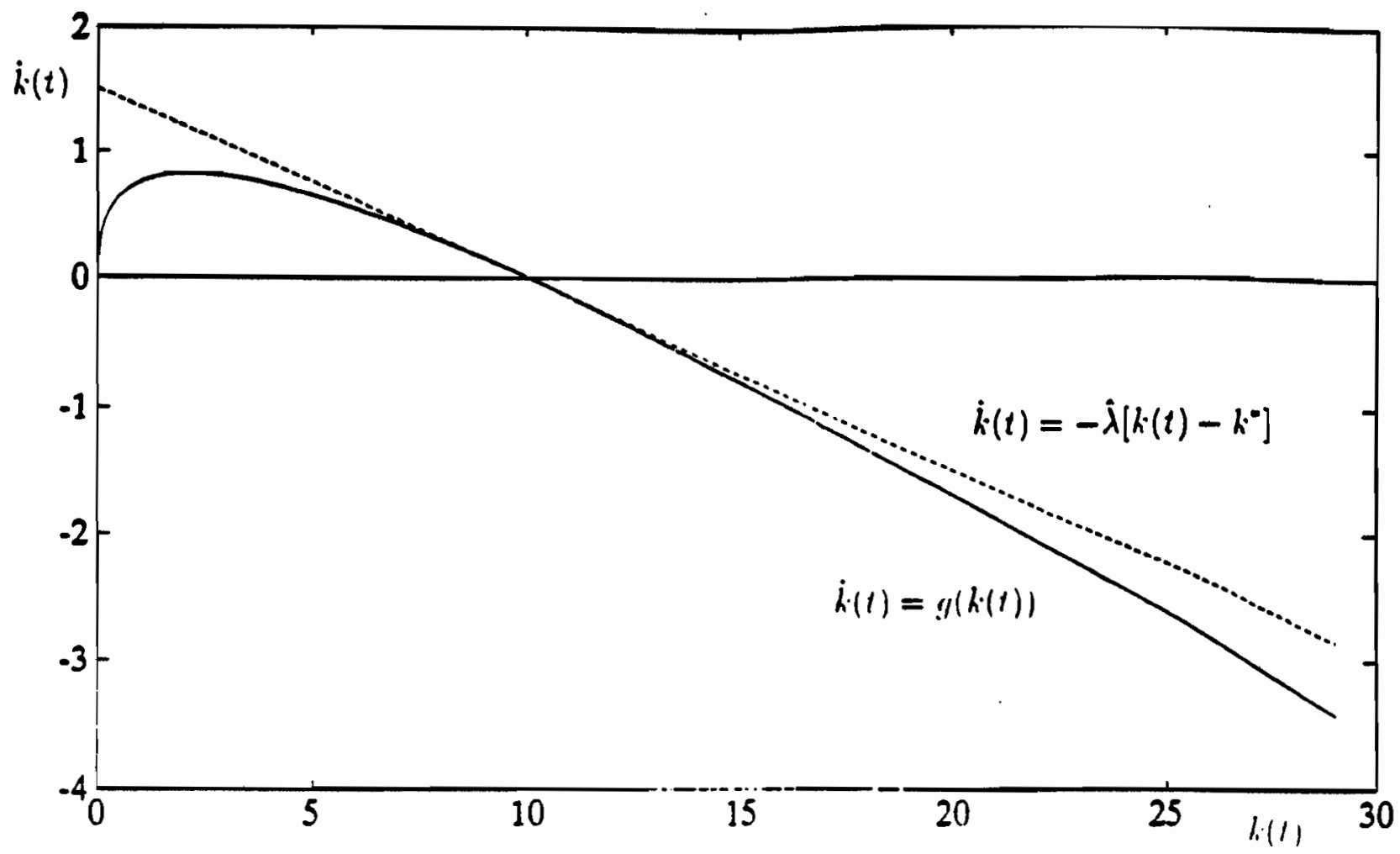

Figure 1(b). Dynamics in the N'coclassical Growth Model: Stable manifolds of the linear and non-linear systems for state variable $k$. Parameter values, $\sigma=0.4, \rho=0.05$, $\beta=0.4, n=0.01, \pi=0.05, A=1$. Steady state, $k^{*}=10.079 .4$. 


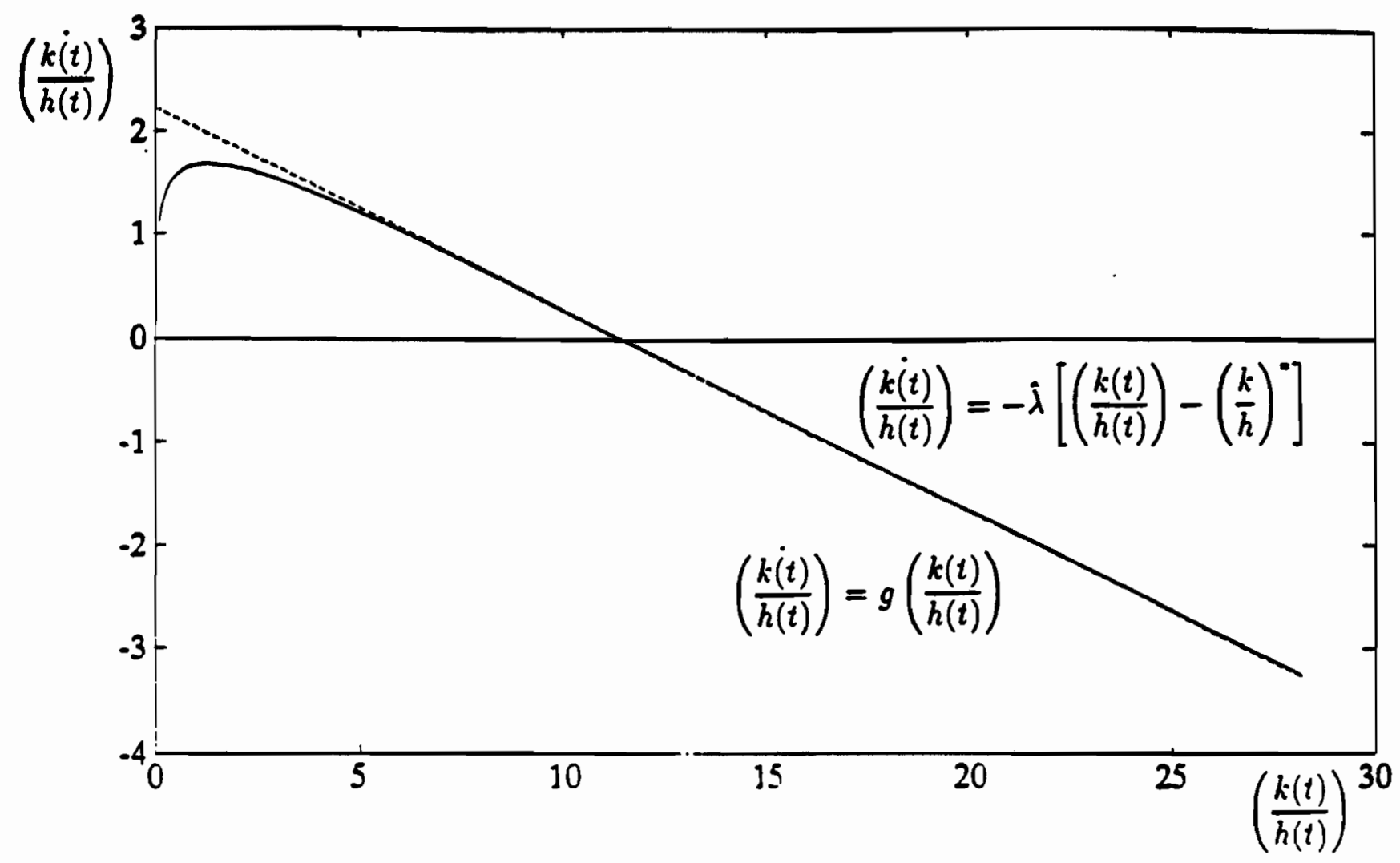

Figure 2(a). Dynamics in the Endogenous Growth Model: Stable manifolds of the linear and non-linear systems for state variable $\left(\frac{k}{h}\right)$. Parameter values, $\delta=0.07$, $\gamma=0.45, \sigma=1.5, \rho=0.05, \beta=0.4, n=0.01, \pi=0.05, \theta=0, A=3$. Steady state, $\left(\frac{k}{h}\right)^{*}=11.4425$. 


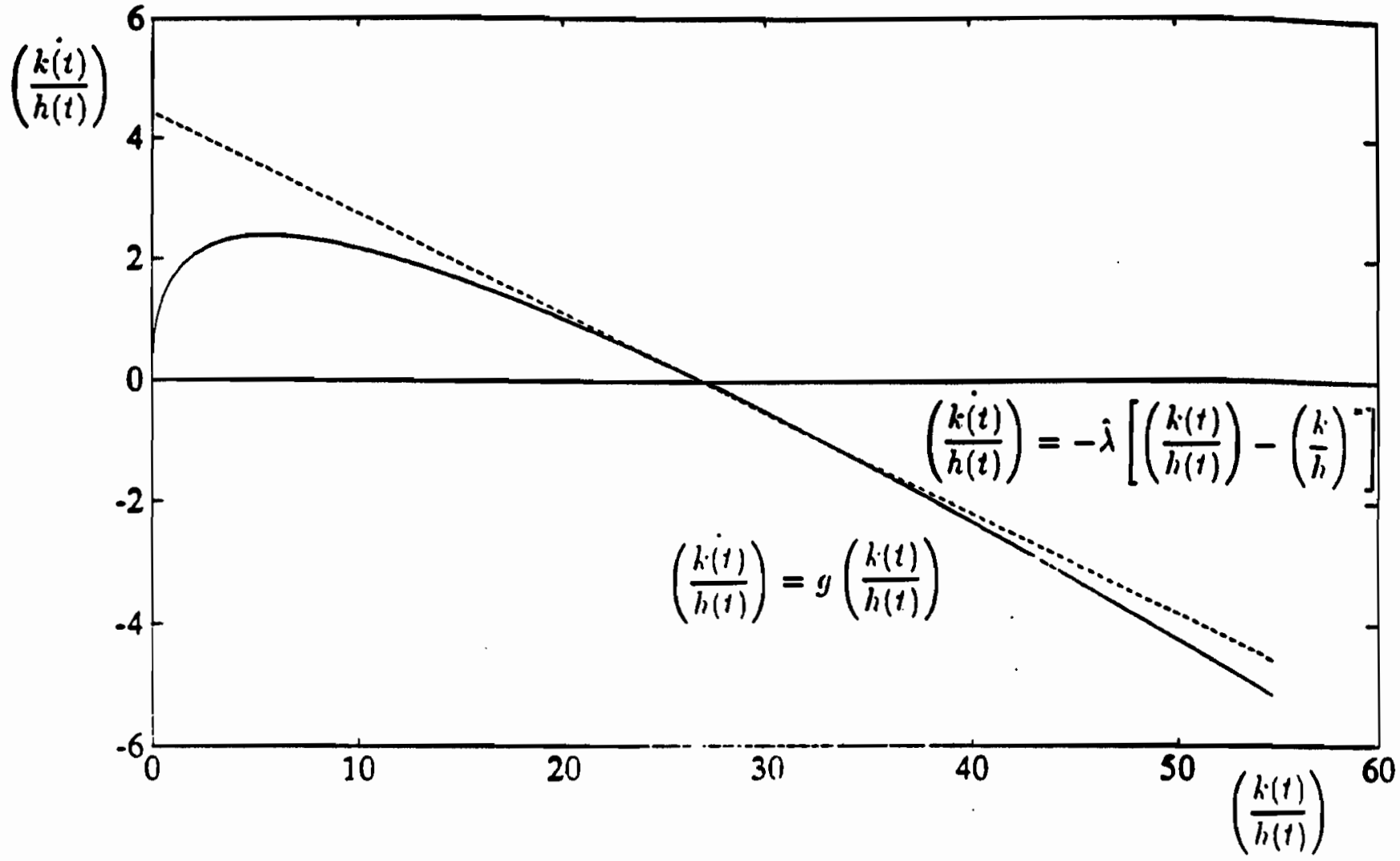

Figure 2(b). Dynamics in the Endogenous Growth Model: Stable manifolds of the linear and non-linear systems for state variable $\left(\frac{k}{h}\right)$. Parameter values, $\delta=0.05, \gamma=1$, $\sigma=0.4, \rho=0.05, \beta=0.4, n=0.01, \pi=0.05, \theta=0, A=3$. Steady state,

$\left(\frac{k}{h}\right)^{\circ}=26.8097$. 